\title{
Demographic Profile and Treatment Outcomes of Filipino Patients with Hepatocellular Carcinoma in a Liver Tumor Registry
}

\author{
Ma. Lourdes O. Daez, Janus P. Ong, Allyn Rey B. Lomboy, Jeffrey M. Libuit, \\ Ivan Michael G. Vicente, Grace C. Firmalino and Gian Carlo A. Carpio \\ Section of Gastroenterology, Department of Medicine, College of Medicine and Philippine General Hospital, University of the Philippines Manila
}

\begin{abstract}
Background and Objectives. Previous studies using older diagnostic criteria indicated chronic Hepatitis B and alcohol as the most common etiologies of HCC in the Philippines. No recent studies using updated criteria for diagnosis have been published. This study used the diagnostic criteria from the latest APASL guidelines to describe the demographic profile of patients with HCC.
\end{abstract}

Methods. This is a cross-sectional study of adult HCC cases from a liver tumor registry in the Philippine General Hospital from 2009 to 2012. Demographics, AFP levels, BCLC stage, Child-Pugh Score, ECOG performance status, treatments received, and mortality were assessed.

Results. The HCC prevalence rate was $7.8 \%$, mostly occurring between ages 40 and 65 years. It is more common in males (M:F=4:1). The most common risk factors are chronic Hepatitis $B$ and alcohol use. Most of the HCC cases were diagnosed at early stages, with less severity of liver functional impairment compared to older studies. Resection was the most common treatment undertaken (50\%) and overall mortality rate at the time of hospital discharge was $25 \%$.

Conclusion. The profile of HCC patients in this study is similar to previous studies. More cases were recognized at earlier stages with better liver function, implying better treatment outcomes with surgery, although selection bias is recognized.

Key Words: hepatocellular carcinoma, Filipino, demographic profile, treatment

Corresponding author: Ma. Lourdes O. Daez, MD

Gastrointestinal Clinic

Philippine General Hospital

Taft Avenue, Ermita, Manila, 1000 Philippines

Fax No.: +6325672983

Email: adetgic@yahoo.com

\section{Introduction}

Hepatocellular carcinoma (HCC) is one of the leading causes of cancer morbidity and mortality in the world. HCC is the sixth most common malignancy worldwide, but it is now the third leading cause of cancer deaths due to its high case fatality rate. ${ }^{1,2}$ Its annual incidence is also increasing, as indicated by a study conducted by the United States Centers for Disease Control; incidence increased to 3.0 per 100,000 in 2006 from 2.7 per 100,000 in 2001. ${ }^{3}$ There are marked geographic differences in its prevalence, reflecting the varied risk factors in different regions of the world. Over $80 \%$ of the world's HCC cases are found in Africa and Asia, where Hepatitis B and C viral infections are still endemic. The incidence rates in developing countries are more than double that found in developed countries. Despite this, the past three decades have seen an increasing incidence in the developed countries probably related to the HCV infection epidemic from the 1970s to the 1990s as well as the observed increase in prevalence of obesity and its relation to the development of nonalcoholic steatohepatitis (NASH) and its sequelae of cirrhosis and HCC. ${ }^{4}$

In the Philippines, a descriptive study in 2009 concluded that the most common risk factors for the development of HCC are chronic Hepatitis B infection and alcoholic beverage intake. However, this study included patients diagnosed with HCC based on histopathologic findings or the presence of a liver nodule greater than two centimeters in size associated with elevated alphafetoprotein (AFP). ${ }^{5}$ Based on the latest guidelines for diagnosis of HCC, the diagnosis can now be made based on the presence of typical findings on dynamic imaging studies without requiring histopathology or an elevated serum AFP.

The treatment of HCC has undergone rapid progress over the past two decades. Improvement in surgical techniques as well as better patient selection and postsurgical care has led to improved patient outcomes. There has been tremendous progress in treatment modalities such as radiofrequency ablation and intra-arterial therapies such as transarterial chemoembolization (TACE) and radioembolization. The first systemic therapy with proven survival benefit was approved in 2008 allowing for the first time treatment of patients with advanced HCC.6,7 


\section{Significance of the Study}

HCC is a disease prevalent in Asia, including the Philippines. This study aimed to describe the risk factors most commonly associated with the development of HCC, and thus the possible impact on public health measures directed at prevention. This study also sought to describe the following:

1. Age, gender

2. Risk factors present

3. Serum AFP levels

4. Barcelona Clinic Liver Cancer (BCLC) stage on presentation

5. Child-Pugh Score on presentation

6. Performance status (ECOG) on presentation

7. Treatment received

A similar study was previously undertaken in the Philippine General Hospital in 2009. However, the current study used different criteria to diagnose HCC. While this current study followed the Asian Pacific Association for the Study of the Liver (APASL) guidelines in diagnosis of HCC, the previous study used the following criteria:

1. Biopsy-proven HCCA

2. Liver nodule $>2 \mathrm{~cm}$ associated with serum AFP $>200 \mathrm{ng} / \mathrm{mL}$

Using the latest guidelines, it is possible to detect patients who were not previously diagnosed with HCC because they did not undergo biopsy or because they had normal AFP levels. This study included such patients and aimed to determine whether any differences in the prevalence of risk factors were present. Also, since new treatment modalities have become available in the Philippines, this study sought to present the outcomes of patients who underwent different treatments.

\section{General Objectives}

1. Determine prevalence of HCC in a liver tumor registry in the Philippine General Hospital

2. Describe the demographic profile of HCC patients in the Philippine General Hospital

3. Determine the interventions received by the patients along with outcomes, where available

\section{Specific Objectives}

1. Determine the prevalence of HCC among patients found to have liver tumors in a liver tumor registry in the Philippine General Hospital

2. Describe the following:

a. Mean age at presentation

b. Gender predilection

c. Associated risk factors

d. Severity of liver disease on diagnosis (Child-Pugh Score)

c. BCLC stage on diagnosis

d. Performance status (ECOG)

3. Present interventions done and determine mortality rate

\section{Methods}

This is a cross-sectional study which included adult patients aged 18 years and above diagnosed with HCC at the Philippine General Hospital from January 2009 to December 2012. Data was retrieved by review of the medical records from the liver tumor registry of the Department of Surgery of said institution. Diagnosis of HCC was confirmed either by histopathology or through the demonstration of typical dynamic radiologic findings (CT or MRI) of early arterial enhancement followed by washout in the portal venous or delayed phases. Diagnosed HCC patients were then characterized based on demographics, presence of risk factors, staging and severity of disease, treatments undertaken, and short-term outcomes of such treatments. Short-term outcomes refer to the status of the patient at the end of the latest admission, as information on subsequent outpatient follow-up for surviving patients was not available.

\section{Results}

A total of 255 patients with hepatic masses were identified based on a search of the computerized surgical database for admitted patients of the Philippine General Hospital. Patient information was thus reviewed and missing data was retrieved from the inpatient records. Out of the 255 patients with liver tumors, 20 were documented to have HCC based on the criteria listed above, which represents $7.8 \%$ of the total number of patients with hepatic masses. The most common diagnosis for liver tumors was metastatic liver disease with 127 cases $(49.8 \%)$. Of these, the most common primary malignancy was colorectal cancer, which made up $55.1 \%$ of the total cases with liver metastases. This was followed by breast cancer and pancreatic cancer, making up $11.0 \%$ and $10.2 \%$ of liver metastases, respectively. Ninety nine (99) cases, representing $38.8 \%$ of the population with hepatic masses, remained without any definite diagnosis up to discharge. The rest of the etiologies of the liver tumors are outlined in Table 1.

Table 1. Etiologies of liver tumors

\begin{tabular}{cc}
\hline \multicolumn{1}{c}{ Diagnosis } & Number (Percentage) \\
\hline Metastatic Liver Disease & $127(49.8 \%)$ \\
Colorectal Cancer & $70(55.1 \%)$ \\
Breast Cancer & $14(11.0 \%)$ \\
Pancreatic Cancer & $13(10.2 \%)$ \\
Unknown primary & $8(6.2 \%)$ \\
Others & $22(17.3 \%)$ \\
Hepatocellular Carcinoma & $20(7.8 \%)$ \\
Cholangiocarcinoma & $4(1.6 \%)$ \\
Benign etiologies & $4(1.6 \%)$ \\
No definite diagnosis & $99(38.8 \%)$ \\
\hline
\end{tabular}

The characteristics of the patients diagnosed with HCC as well as mode of diagnosis, presence of risk factors, and presenting symptom are described in Table 2 . The mean age of patients at diagnosis of HCC was 54.42 years with a male 
preponderance (M:F=4:1). More patients were diagnosed with HCC based on histopathology rather than radiologic findings (70\% vs. 30\%, respectively), likely due to the source of the data being a surgical database. Most records did not report any identifiable risk factors for HCC, but of the reported risk factors, the most common is chronic Hepatitis B infection (25\%) followed by a combination of chronic Hepatitis B and alcohol use (10\%). All of the chronic Hepatitis B patients were HBeAg negative. None of the HCC patients had immunization against Hepatitis $B$. The most common presenting symptom is abdominal pain $(64.3 \%)$ followed by abdominal enlargement (14.3\%).

Table 2. Demographic data of patients with confirmed HCC

\begin{tabular}{ll}
\hline Age (years) & $(\mathrm{N}=20)$ \\
$<40$ & $5(25 \%)$ \\
$40-65$ & $12(60 \%)$ \\
$>65$ & $3(15 \%)$ \\
Mean & $54.42 \pm 13.20$ \\
& \\
Gender & $(\mathrm{N}=20)$ \\
Males & 16 \\
Females & 4 \\
Male:Female ratio & $4: 1$ \\
& \\
Mode of Diagnosis & $(\mathrm{N}=20)$ \\
Characteristic findings on dynamic imaging & $6(30 \%)$ \\
Histopathology & $14(70 \%)$ \\
& \\
Risk factors present & $(\mathrm{N}=20)$ \\
Chronic Hepatitis B & $5(25 \%)$ \\
Alcohol intake & $1(5 \%)$ \\
Chronic Hepatitis B + alcohol & $2(10 \%)$ \\
No definite risk factors identified & $12(60 \%)$ \\
& \\
Hepatitis serology & $(\mathrm{N}=12)$ \\
Active Hepatitis B, eAg (+) & 0 \\
Active Hepatitis B, eAg $(-)$ & $7(58.3 \%)$ \\
Remote/Occult Hepatitis B & $1(8.3 \%)$ \\
Resolved Hepatitis B & $3(25.0 \%)$ \\
Immunized for Hepatitis B & 0 \\
Presentation & $1(7.1 \%)$ \\
Abdominal pain &
\end{tabular}

The mean size of the tumors was $8.5 \mathrm{~cm}$, most measuring greater than $5 \mathrm{~cm}(61.5 \%)$. Of the cases in which the number of tumors was reported, $62.5 \%$ had a single tumor, while $25 \%$ had three or more tumors. None had demonstrable distant metastases on presentation. Most patients had unilobar involvement $(76.5 \%)$. Although the mean tumor size was large, the rest of the tumor characteristics were consistent with favorable surgical outcomes. Of the cases with reported serum AFP levels, a greater number of patients had values less than the cut-off for diagnosis of HCC compared to those with elevated values, with $66.7 \%$ having serum AFP values $<200 \mathrm{ng} / \mathrm{mL}$. Unfortunately, more than half of the patients in this study did not have serum AFP determinations, but the higher frequency of HCC cases with non-elevated serum AFP as well as the decreased utilization of the test even in a large tertiary center such as PGH may further support the recommendation that serum AFP alone is not a reliable diagnostic for HCC (APASL). These findings are detailed in Table 3.

Table 3. Tumor characteristics and serum AFP levels of HCC patients $(\mathrm{N}=$ number of records with a value reported)

\begin{tabular}{cll}
\hline $\begin{array}{c}\text { Tumor characteristics } \\
\text { Size (mean, in cm) }\end{array}$ & $(\mathrm{N}=13)$ & 8.5 \\
Tumors $<2 \mathrm{~cm}$ & & \multicolumn{1}{l}{ None } \\
Tumors $2-5 \mathrm{~cm}$ & & $5(38.5 \%)$ \\
Tumors $>5 \mathrm{~cm}$ & & $8(61.5 \%)$ \\
Single & $(\mathrm{N}=16)$ & \\
2 & & $10(62.5 \%)$ \\
3 or more & & $2(12.5 \%)$ \\
Number of tumors & $4(25 \%)$ \\
One lobe & $(\mathrm{N}=17)$ & $13(76.5 \%)$ \\
Both lobes & & $4(23.5 \%)$ \\
Lobar involvement & $(\mathrm{N}=9)$ & $6(66.7 \%)$ \\
Serum AFP levels (in ng/mL) & & 0 \\
$<200 \quad$ & & $3(33.3 \%)$ \\
$200-1000$ & & None \\
\hline $1000 \quad$ & & \\
Metastases
\end{tabular}

Table 4 illustrates the distribution of cases according to different stages of HCC (BCLC staging), severity of liver impairment (Child-Pugh score), and performance status (ECOG). With respect to BCLC stage, most patients $(64.3 \%)$ were diagnosed in the earlier stages of disease (Stage 0 to B) which would represent candidates for active therapy, in contrast to BCLC stage D patients for whom best supportive care only would be most appropriate. The majority of patients had a Child-Pugh score of B (45.5\%) followed by Child-Pugh A (36.4\%). Only $18.2 \%$ of patients had severe liver impairment (Child-Pugh C), generally excluding them from interventions with curative intent. Also, most patients had good to fair performance status from ECOG 0-2 (92.3\%) compared to those with ECOG 3-4 (7.7\%) which would be the population for which only best supportive care is appropriate based on the BCLC algorithm. Taking this information into consideration along with the information presented in Table 3, most HCC patients in this registry were good candidates for active therapies including curative strategies such as resection, transplantation and radiofrequency ablation, as well as palliative modalities such as TACE or Sorafenib.

Table 5 shows that most of the patients underwent curative resection $(58.8 \%)$ which is consistent with the data presented in the previous tables demonstrating the eligibility of most of the patients in this database for surgery. It is interesting that based on the BCLC staging system only three patients in this study should be eligible for resection. However, it is a known fact that in Asia, the presence of 
large $(>5 \mathrm{~cm})$ or multifocal lesions does not preclude consideration for resection. ${ }^{8} \mathrm{~A}$ number of patients $(17.6 \%)$ did not receive any treatment at all, although the reasons for this are varied, such as the medical condition of the patient, unavailability of funds, and patient preference. There were five deaths in this study, representing $25 \%$ of the population with HCC. Three deaths were probably related to the HCC itself while the other two appeared unrelated to the HCC (upper GI bleed from bleeding peptic ulcer, acute myocardial infarction).

Table 4. Distribution of HCC stage and severity of liver disease

\begin{tabular}{cll}
\hline BCLC stage & $(\mathrm{N}=14)$ & \\
0 & & 0 \\
A & & $3(21.4 \%)$ \\
B & & $6(42.9 \%)$ \\
C & $3(21.4 \%)$ \\
D & $2(14.3 \%)$ \\
Child-Pugh Score & $(\mathrm{N}=11)$ & \\
A & & $4(36.4 \%)$ \\
B & & $5(45.5 \%)$ \\
C & $2(18.2 \%)$ \\
ECOG & $(\mathrm{N}=13)$ & \\
0 & & $8(61.5 \%)$ \\
$1-2$ & & $4(30.8 \%)$ \\
$3-4$ & & $1(7.7 \%)$ \\
\hline
\end{tabular}

Table 5. Treatments administered, mortality rate and causes of death

\begin{tabular}{ll}
\hline Treatments Undertaken & $(\mathrm{N}=17)$ \\
$\quad$ Resection & $10(58.8 \%)$ \\
TACE & $3(17.6 \%)$ \\
Sorafenib & $1(5.9 \%)$ \\
None & $3(17.6 \%)$ \\
Short-term mortality rate & 5 deaths $(25 \%)$ \\
$\begin{array}{l}\text { Causes of mortality } \\
\text { probably HCC-related (variceal bleed, sepsis, }\end{array}$ & $3(60 \%)$ \\
$\begin{array}{l}\text { HCC progression) } \\
\text { probably HCC-unrelated }\end{array}$ & $2(40 \%)$ \\
\hline
\end{tabular}

In 2010, the APASL published consensus recommendations on the management of hepatocellular carcinoma. Included in its recommendations is the use of characteristic findings on dynamic CT or imaging studies, specifically the demonstration of early arterial enhancement of a liver tumor with contrast washout in the portal venous or delayed phases, to diagnose HCC without the need for histopathologic confirmation. ${ }^{8}$ This study made use of this criterion as well as histopathology in the detection of patients with HCC from a surgical database.

In this study, the prevalence of HCC was computed at $7.8 \%$, although this is likely an underestimation as $38.8 \%$ of the patients in the registry did not have a final diagnosis even upon discharge. The most common diagnosis of the liver tumors was metastatic liver disease, with colorectal cancer comprising the majority.

A study conducted at the Philippine General Hospital in 2009 by Lucas et al. also looked at the profile of HCC patients with similar demographics, although said study made use of old diagnostic criteria. The mean age at diagnosis from Lucas's study was 54, similar to the mean age derived from this study (54.42). The male-to-female ratio in the previous study was 3.5:1, nearly same as the ratio of patients in this study (4:1). The most common chief complaint in both studies was abdominal pain, and the most common risk factors (chronic hepatitis B and alcohol use) identified in both studies were the same. However, the two studies differed in terms of distribution of patients across the different BCLC stages. The earlier study reported that $87.8 \%$ of HCC patients were in the advanced stage and $11.7 \%$ had terminal disease. In contrast, since this current study made use of a surgical database, there is an inherent bias in selection because patients referred to the Surgery Service were likely deemed fit for surgical intervention prior to the referral or admission. Hence, most of the patients in this study were in the earlier stages of HCC. In terms of treatment, most of the patients from the earlier study did not undergo any treatment at all, as opposed to the patients in this study, most of whom underwent tumor resection. Another difference is that the older study reported therapies such as tamoxifen and systemic chemotherapy which are no longer recommended by the latest guidelines. Sorafenib, the only systemic therapy with proven survival benefit for advanced hepatocellular carcinoma, ${ }^{9}$ was used in one patient in the current study whereas it was not yet available at the time of the earlier study. ${ }^{5}$

Except for tumor size, the reported tumor characteristics in this study all point to favorable outcomes following surgery. Interestingly, despite the large mean size of the tumors in this study, most of the cases with serum AFP determinations reported levels $<200 \mathrm{ng} / \mathrm{mL}$ which is the recommended cut-off for diagnosis of HCC. ${ }^{8}$ This finding lends weight to the recommendation by the APASL that serum AFP should not be used alone in diagnosing HCC, as this may cause clinicians to miss potentially curable tumors.

Most of the patients in this study were diagnosed at early stages of HCC (BCLC 0-B), with fair liver function (Child-Pugh A and B) and good to fair performance status $(0-2)$. Curative resection was undertaken in most of the patients, although a significant percentage underwent TACE and no treatment at all. While the overall mortality rate was $25 \%$, an association among stage and severity of liver disease, treatment, and mortality could not be ascertained due to the small population size. Despite this limitation, it is suggested by the findings in this study that most of the 
patients referred for surgical intervention are in fact appropriate candidates for therapies with curative intent. ${ }^{10}$

This study is limited by its retrospective nature. Since data collection relied on review of medical records, the available data for each patient was very variable. Because the APASL guidelines for diagnosis were used, tumors which were already previously being treated as HCC but did not fulfill the criteria or did not report basis for the diagnosis were excluded. This explains the small population size and the potentially underestimated HCC prevalence found in this study. In addition, the small population size made statistical analysis for associations between different factors and mortality inadequate.

\section{Conclusion}

The profile of HCC patients from the liver tumor registry used in this study was similar to that reported in previous studies. However, HCC patients seen by the Surgery Service were more likely to have early stage disease with fair liver function and good functional status. These characteristics make curative resection the treatment of choice for the majority of these patients which, correspondingly, is the most common treatment undertaken in this study. A large prospective study using the latest guidelines for the diagnosis of HCC involving surgical as well as non-surgical HCC patients needs to be undertaken to reliably determine the associations among severity of disease, treatment, and eventual outcomes.

\section{References}

1. Venook AP, Papandreou C, Furuse J, de Guevaral LL. The incidence and epidemiology of hepatocellular carcinoma: a global and regional perspective. Oncologist. 2010; 15 Suppl 4:5-13. 15:5-13.

2. Altekruse SF, McGlynn KA, Reichman ME. Hepatocellular carcinoma incidence, mortality, and survival trends in the United States from 1975 to 2005. J Clin Oncol. 2009; 27(9):1485-91.

3. O'Connor S, Ward JW, Watson M, Momin B, Richardson LC, Centers for Disease Control and Prevention (CDC). Hepatocellular Carcinoma United States, 2001-2006. MMWR Morb Mortal Wkly Rep. 2010; 59(17):517-20.

4. Yang JD, Roberts LR. Hepatocellular carcinoma: A global view. Nat Rev Gastroenterol Hepatol. 2010; 7(8):448-58.

5. Lucas ZDF, Pangan CP, Patal PC, Ong JP. The clinical profile of hepatocellular carcinoma patients at the Philippine General Hospital. Philipp J Intern Med. 2009; 47:1-9.

6. Lencioni $R$, Chen XP, Dagher L, Venook AP. Treatment of intermediate/advanced hepatocellular carcinoma in the clinic: how can outcomes be improved? Oncologist. 2010; 15 Suppl 4:42-52.

7. Fernando J, Sancho P, Rodriguez CM, et al. Sorafenib Sensitizes Hepatocellular Carcinoma Cells to Physiological Apoptotic Stimuli. J Cell Physiol. 2012; 227(4):1319-25.

8. Omata M, Lesmana LA, Tateishi R, et al. Asian Pacific Association for the Study of the Liver consensus recommendations on hepatocellular carcinoma. Hepatol Int. 2010; 4(2):439-74.

9. Llovet JM, Ricci S, Mazzaferro V, et al. Sorafenib in advanced hepatocellular carcinoma. N Engl J Med. 2008; 359(4):378-90.

10. Waly Raphael S, Yangde Z, YuXiang C. Hepatocellular carcinoma: focus on different aspects of management. ISRN Oncol. 2012; 2012:421673. 